\title{
Boundary layer flow over a moving plate in MHD Jeffrey nanofluid: A revised model
}

\author{
$S M Z_{\text {Zokri }}{ }^{1}, N S$ Arifin $^{1}, A R M \mathrm{Kasim}^{1}, N F$ Mohammad $^{2}$, and $M Z$ Salleh $^{1, *}$ \\ ${ }^{1}$ Faculty of Industrial Science \& Technology, Universiti Malaysia Pahang, 26300 UMP Kuantan, \\ Pahang, Malaysia \\ ${ }^{2}$ Dept. of Comp. Theo. Sc., Kulliyyah of Science, International Islamic University Malaysia, 25200 \\ Kuantan, Pahang, Malaysia
}

\begin{abstract}
The flow and heat transfer of magnetohydrodynamic (MHD) Jeffrey nanofluid induced by a moving plate is examined numerically. The formulation is established by using the revised model of passively controlled boundary layer instead of actively, which is more realistic physically. The similarity transformation variables are used to transform the partial differential equations into a set of ordinary differential equations before solving it via numerical approach called as the Runge-Kutta Fehlberg method. Graphical representation of the physical parameters over the temperature profile is deliberated. Temperature profile is slowed down due to the parameters of Deborah number and plate velocity while the reverse trend is observed for thermophoresis diffusion parameter. The Brownian motion has shown an insignificant outcome on the temperature profile. A comparison with the earlier publication has been conducted and a perfect agreement between the data is detected.
\end{abstract}

\section{Introduction}

In the recent decades, non-Newtonian fluids have been widely used in many industrial applications due to its multi-component and chemically complex as well as displaying different degrees of elasticity, viscosity and thixotropy shear dependence [1]. Among the non-Newtonian fluids, a simple yet elegant Jeffrey fluid model is taken into consideration for its capability of depicting both the relaxation and retardation parameters, which has never been issued in existing models [2]. However, due to the restriction in its thermal conductivity, this fluid model is not appropriate to reach a better industrial cooling rate. To overcome this weakness, a working fluid namely nanofluid is integrated in the base fluid (Jeffrey fluid) with the aim of enhancing the thermal performance. Particularly, a small amount of nanoparticles $(<1 \%)$ added into the conventional fluid has significantly improved about $10-20 \%$ of its thermal conductivity [3].

The boundary layer flow on a constant speed moving plate was first reported by Sakiadis [4]. An experimental study was later conducted by Tsou et al. [5] to support the Sakiadis' theoretical predictions. A number of investigations has been performed since then on the moving plate, for instance by Elbashbeshy and Bazid [6], Weidman et al. [7],

*Corresponding author: zuki@ump.edu.my 
Bachok et al. [8], Ishak et al. [9], etc. Using the Buongiorno model, Roşca and Pop [10] examined the parameters of Brownian motion and thermophoresis diffusion from a moving surface in an external uniform free stream. The influence of viscous dissipation in a nanofluid towards a moving plate was studied by Mohamed et al. [11]. Their study has exposed that the moving effect had strongly reduced the temperature profile along with nanoparticle volume fraction. More recently, the stability of the dual solutions for nanofluid model was discovered by Jahan et al. [12] over a moving surface. In view of stability analysis, positive smallest eigenvalues give rise to the upper branch solution, while negative eigenvalues yield the lower branch solution. Therefore, the solution for the upper branch is stable and physically achievable.

The objective of the present work is to examine the realistic passive boundary condition on the boundary layer flow over a moving plate immersed in Jeffrey nanofluid numerically. Here, the condition of zero normal flux of nanoparticles at the plate that incorporates the impacts of Brownian motion and thermophoresis parameters is explained to drive away the particles from the wall $[13,14]$. It is a sizable upgrading of boundary conditions proposed by Kuznetsov and Nield [15] where the nanoparticle volume fraction at the wall passively corrects itself to whatever temperature executed on the surface and thus, the effect of Buongiorno's model can be implemented in a more effective approach. Thus far, the numerical results accessible in this study are professed as new as it has never been published elsewhere.

\section{Mathematical formulation}

A steady, two-dimensional and laminar flow of a moving plate embedded in MHD Jeffrey nanofluid with a passive controlled model is inspected. The plate velocity is denoted as $u_{w}(x)=\varepsilon U_{\infty}$, where $\varepsilon$ and $U_{\infty}$ being the plate velocity parameter and free stream velocity, respectively. Here, $\varepsilon=0$ denotes the free convection flow, $\varepsilon>0$ implies the downstream movement of the plate from the origin, whereas $\varepsilon<0$ signifies the upstream movement of the plate towards the origin [7]. Let $T, T_{w}$ and $T_{\infty}$ be the respective boundary layer, wall and ambient temperature while $C, C_{w}$ and $C_{\infty}$ being the nanoparticle volume fraction, nanoparticle volume fraction at surface and ambient nanoparticle volume fraction. A constant magnetic field, $B_{0}$ is applied vertically to the plate, as displayed in the flow diagram of fig. 1 . Under these conditions, the governing boundary layer equations are [2, 11 .

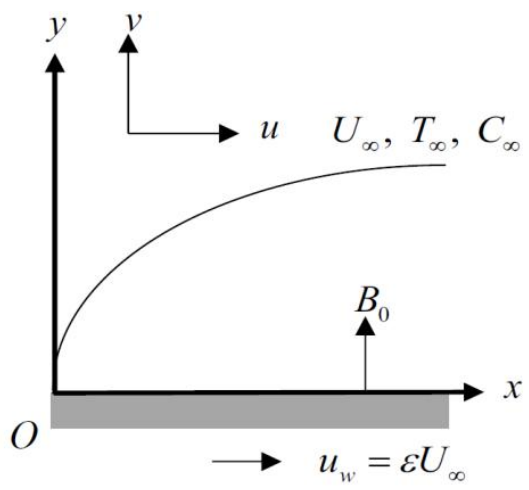

Fig. 1. The flow diagram. 


$$
\begin{gathered}
\frac{\partial u}{\partial x}+\frac{\partial v}{\partial y}=0 \\
u \frac{\partial u}{\partial x}+v \frac{\partial u}{\partial y}=\frac{v}{1+\lambda}\left[\frac{\partial^{2} u}{\partial y^{2}}+\lambda_{1}\left(u \frac{\partial^{3} u}{\partial x \partial y^{2}}-\frac{\partial u}{\partial x} \frac{\partial^{2} u}{\partial y^{2}}+\frac{\partial u}{\partial y} \frac{\partial^{2} u}{\partial x \partial y}+v \frac{\partial^{3} u}{\partial y^{3}}\right)\right]-\frac{\sigma B_{0}^{2}}{\rho_{f}} u \\
u \frac{\partial T}{\partial x}+v \frac{\partial T}{\partial y}=\alpha \frac{\partial^{2} T}{\partial y^{2}}+\tau\left[D_{B} \frac{\partial C}{\partial y} \frac{\partial T}{\partial y}+\frac{D_{T}}{T_{\infty}}\left(\frac{\partial T}{\partial y}\right)^{2}\right] \\
u \frac{\partial C}{\partial x}+v \frac{\partial C}{\partial y}=D_{B} \frac{\partial^{2} C}{\partial y^{2}}+\frac{D_{T}}{T_{\infty}} \frac{\partial^{2} T}{\partial y^{2}}
\end{gathered}
$$

with the boundary conditions

$$
\begin{array}{r}
u=u_{w}(x)=\varepsilon U_{\infty}, v=0, T=T_{w}, D_{B} \frac{\partial C}{\partial y}+\frac{D_{B}}{T_{\infty}} \frac{\partial T}{\partial y}=0 \text { at } y=0 \\
u \rightarrow U_{\infty}, v \rightarrow 0, T \rightarrow T_{\infty}, C \rightarrow C_{\infty} \text { as } y \rightarrow \infty
\end{array}
$$

where $u$ and $v$ are the respective components of velocity in the $x$ - and $y$-directions, $v$ is the kinematic viscosity, $\lambda$ and $\lambda_{1}$ are the respective ratio of relaxation to retardation times and retardation time, $\rho_{f}$ is the density for the base fluid, $\sigma$ is the electrically conductivity, $\alpha=k /(\rho C)_{f}$ is the thermal diffusivity, $\tau=(\rho c)_{p} /(\rho c)_{f}$ is the ratio of heat capacity where $(\rho c)_{p}$ is the heat capacity of the nanoparticle while $(\rho c)_{f}$ is the heat capacity of the fluid, $k$ is the thermal conductivity, $D_{B}$ is the Brownian diffusion coefficient and $D_{T}$ is the thermophoretic diffusion coefficients. The boundary condition of $D_{B} \frac{\partial C}{\partial y}+\frac{D_{B}}{T_{\infty}} \frac{\partial T}{\partial y}=0$ embodies the normal flux of nanoparticles is zero [15]. Introducing the set of similarity transformation variables [8],

$$
\eta=\left(\frac{U_{\infty}}{2 x v}\right)^{1 / 2} y, \quad \psi=\left(2 U_{\infty} x v\right)^{1 / 2} f(\eta), \quad \theta(\eta)=\frac{T-T_{\infty}}{T_{w}-T_{\infty}}, \quad \phi(\eta)=\frac{C-C_{\infty}}{C_{\infty}}
$$

In the above equations, the $\eta, \theta$ and $\phi$ are the respective similarity variable, dimensionless temperature of the fluid and rescaled nanoparticle volume fraction. The stream function, $\psi$ which is defined as $u=\partial \psi / \partial y$ and $v=-\partial \psi / \partial x$ has automatically satisfied equation(1). Specifically, $u$ and $v$ can be written as follows

$$
u=U_{\infty} f^{\prime}(\eta), \quad v=-\left(\frac{U_{\infty} v}{2 x}\right)^{\frac{1}{2}} f(\eta)+\frac{U_{\infty} y}{2 x} f^{\prime}(\eta)
$$

Substituting equations (6) and (7) into equations (2) to (5), the resulting ordinary differential equations are

$$
\begin{aligned}
f^{\prime \prime \prime}-\frac{\lambda_{2}}{2} f f^{(i v)}+(1+\lambda)\left(f f^{\prime \prime}-2 M f^{\prime}\right) & =0 \\
\frac{1}{\operatorname{Pr}} \theta^{\prime \prime}+f \theta^{\prime}+N b \theta^{\prime} \phi^{\prime}+N t \theta^{\prime 2} & =0
\end{aligned}
$$




$$
\begin{array}{r}
\phi^{\prime \prime}+L e \operatorname{Pr} f \phi^{\prime}+\frac{N t}{N b} \theta^{\prime \prime}=0 \\
f(0)=0, f^{\prime}(0)=\varepsilon, \theta(0)=1, \quad N b \phi^{\prime}(0)+N t \theta^{\prime}(0)=0 \\
f^{\prime}(\infty) \rightarrow 1, \quad f^{\prime \prime}(\infty) \rightarrow 0, \quad \theta(\infty) \rightarrow 0, \quad \phi(\infty) \rightarrow 0
\end{array}
$$

where $\lambda_{2}=\frac{\lambda_{1} U_{\infty}}{x}, M=\frac{x \sigma B_{0}^{2}}{U_{\infty} \rho_{f} a}, \operatorname{Pr}=\frac{v}{\alpha}, N b=\frac{\tau D_{B} C_{\infty}}{v}, N t=\frac{\tau D_{T}\left(T_{w}-T_{\infty}\right)}{v T_{\infty}}, L e=\frac{a}{D_{B}}$ are the respective Deborah number, magnetic parameter, Prandtl number, Brownian motion parameter, thermophoresis parameter and Lewis number.

\section{Results and discussion}

Equations (8) to (10) with boundary conditions (11a) and (11b) were solved numerically using the Runge-Kutta Fehlberg method with the help of Maple software. Parameters of Deborah number $\lambda_{2}$, plate velocity parameter $\varepsilon$, Brownian motion $N b$ and thermophoresis parameter $N t$ are tackled over the temperature profile. For validation purposes, the comparison among the present study with the earlier publications reported by Roşca and Pop [10] and Mohamed et al. [11] is carried out for several values of Pr, i.e. $\operatorname{Pr}=0.7,0.8,1,5,10$. Apparently, the numerical values in table 1 are excellently closed to each other; hence, the numerical code utilised throughout this study is concluded to effectually solve the current problem. Referring to Mohamed et al. $[11,16], \operatorname{Pr}=7$ is used for the current numerical simulations as it represents water that usually acts as the base fluid for nanofluid.

Table 1. Comparison values of $-\theta^{\prime}(0) / \sqrt{2}$ for several values of $\operatorname{Pr}$ when

$$
\varepsilon=\lambda=\lambda_{2}=N b=N t=L e=0 .
$$

\begin{tabular}{|c|c|c|}
\hline $\operatorname{Pr}$ & Mohamed et al. [11] & Present \\
\hline 0.7 & 0.292680 & 0.292997 \\
\hline 0.8 & 0.306917 & 0.307240 \\
\hline 1 & 0.332057 & 0.332381 \\
\hline 5 & 0.576689 & 0.576683 \\
\hline 10 & 0.728141 & 0.728140 \\
\hline
\end{tabular}

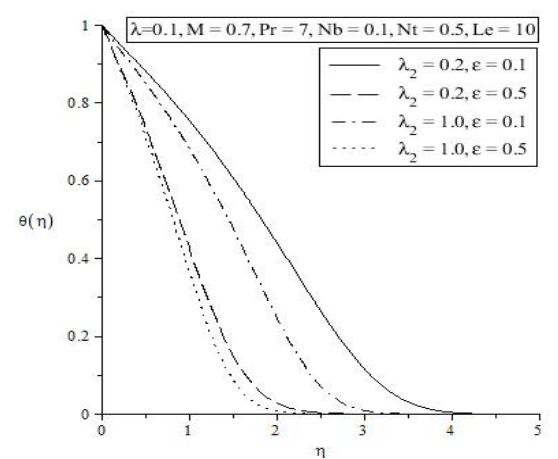

Fig. 2. $\theta(\eta)$ against $\eta$ for several values of $\lambda_{2}$ and $\varepsilon$.

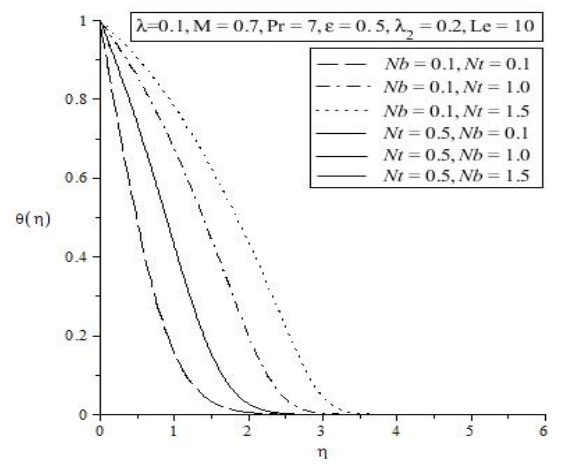

Fig. 3. $\theta(\eta)$ against $\eta$ for several values of $N b$ and Nt. 
The temperature profile $\theta(\eta)$ versus $\eta$ for both parameters of Deborah number $\lambda_{2}$ and plate velocity $\varepsilon$ are disclosed in fig. 2 . With the increase of $\lambda_{2}$ and $\varepsilon$, the reduction of $\theta(\eta)$ and the thickness of thermal boundary layer is detected. Parameter $\lambda_{2}$ is functioned to measure the viscoelasticity of the materials. Increasing $\lambda_{2}$ means the material performs quite elastically while at the same time reinforcing the fluid friction. Due to the frictional force, heat is engaged in the flow longer rather than transferring it to the surrounding. The attendance of parameter $\varepsilon$, on the other hand tends to passage away the fluid from the plate region speedily, so do the heat owing to the augmentation of the ratio of velocity differences between the plate and fluid. Consequently, the thermal diffusivity is lessening, and the declination of the temperature field is perceived.

Fig. 3 revealed the impact of thermophoresis diffusion $N t$ and Brownian motion $\mathrm{Nb}$ parameters on the temperature profile. It is noted that the temperature field is amplified due to the reponse of $N t$. Higher $N t$ has surged up the temperature differences, thereby strengthening the dispersion of nanoparticles towards the cooler environment. Meanwhile, the effect of $\mathrm{Nb}$ is not dominant over the temperature field as the insignificant outcome is detected, mainly reasoned by the zero normal flux at the plate (passive controlled model). This result is in accordance with the results documented by Halim et al. [13] and Rehman et al. [14].

\section{Conclusion}

Present study examines the boundary layer flow of MHD Jeffrey nanofluid passing through a moving plate with the passive controlled model. In the presence of nanoparticles, the thermal diffusivity of Jeffery fluid is enhanced. The implementation of passive controlled model is more physically realistic in comparison to the active controlled model. The effect of Jeffrey fluid parameter $\left(\lambda_{2}\right)$ together with nanofluid parameters ( $N b$ and $N t$ ) are thoroughly investigated on the temperature field. The following conclusions can be drawn from this study:

- Temperature profile is decreased due to increasing parameters $\lambda_{2}$ and $\varepsilon$.

- Temperature profile is increased because of increasing $N t$.

- The effect of $\mathrm{Nb}$ on the temperature field is insignificant.

\section{Acknowledgement}

The authors are appreciative to the provisions received from Universiti Malaysia Pahang (UMP) (PGRS1703100 and RDU170358).

\section{References}

1. Pearson J and Tardy P 2002 Journal of Non-Newtonian Fluid Mechanics 102 447-73

2. Zokri S M, Arifin N S, Mohamed M K A, Salleh M Z, Kasim A R M and Mohammad N F 2017 Malaysian Journal of Fundamental and Applied Sciences 13 279-84

3. Choi S, Zhang Z, Yu W, Lockwood F and Grulke E 2001 Applied Physics Letters 79 2252-4

4. Sakiadis B 1961 AIChE Journal 7 26-8 
5. Tsou F, Sparrow E and Goldstein R J 1967 International Journal of Heat and Mass Transfer 10 219-35

6. Elbashbeshy E and Bazid M 2000 Journal of Physics D: Applied Physics 332716

7. Weidman P, Kubitschek D and Davis A 2006 International Journal of Engineering Science 44 730-7

8. Bachok N, Ishak A and Pop I 2010 International Journal of Thermal Sciences 49 1663-8

9. Ishak A, Yacob N A and Bachok N 2011 Meccanica 46 795-801

10. Roşca N C and Pop I 2014 Computers \& Fluids 95 49-55

11. Mohamed M, Noar N, Salleh M and Ishak A 2016 Journal of Applied Fluid Mechanics 9

12. Jahan S, Sakidin H, Nazar R and Pop I 2017 International Journal of Mechanical Sciences 131-132 1073-81

13. Halim N, Haq R U and Noor N 2017 Meccanica 52 1527-39

14. Rehman S-u, Haq R-u, Khan Z H and Lee C 2016 Journal of the Taiwan Institute of Chemical Engineers 63 226-35

15. Kuznetsov A and Nield D 2013 International Journal of Heat and Mass Transfer 65 682-5

16. Mohamed M K A, Noar N A Z M, Salleh M Z and Ishak A 2016 Sains Malaysiana 45 289-96 